\title{
Phase Measurement Method for Medium Voltage Power Line Channel Based on GPS
}

\author{
Zhiyuan Xie \& Xiao Zhang \\ School of Department of electronics and Communication Engineering, North China Electric Power \\ University, Baoding 071000, China \\ Lichong Wang \\ Hebei Shenke electronic limited by Share Ltd, Shijiazhuang 050000, China
}

\begin{abstract}
Medium voltage power line communication has attracted the attention of researchers for its unique advantages. But it has been very little research on the frequency characteristics of medium voltage power line channel for a long time. Proposed based GPS remote synchronization zero-IF quadrature demodulation phase measurement method, which can effectively solve the problem of the phase measurement of the medium voltage power line. Paper introduces the basic principle of this method, and through the simulation of two cases the ideal channel and the RC channel, fully verifies the correctness and feasibility of the method.
\end{abstract} KEYWORD: Medium voltage power line; channel characteristics; GPS

\section{INTRODUCTION}

Medium voltage power line communication with a wide distribution, infrastructure facilities and low cost advantages, especially for sparsely populated residential population dispersion and other difficult to lay optical fiber or optical fiber laying high-cost rural and mountainous areas have high practical value (S. Xu et al, 2010). Due to the medium voltage power line channel as the major part of the medium voltage power line communication, the effects of its own characteristics of communication reliability cannot be ignored, so testing the characteristics of the medium voltage power line channel is particularly important.

For in-depth study of medium voltage power line communication modulation and coding of key technologies, study medium voltage power lines channel transmission characteristics is of great significance. At present, the test method of power line channel is mainly to test the attenuation characteristic of the channel. But these methods can only measure the attenuation of the channel "amplitude-frequency characteristics" and cannot be tested channel "phasefrequency characteristics." The phase-frequency characteristics of the channel are very important for some design of the modulation mode. So it is very important to find a method to measure the amplitude-frequency response and phase -frequency response of medium voltage power line at the same time.

\section{ZERO-IF QUADRATURE DEMODULATION METHOD BASED ON GPS}

Phase measurement has a wide range of applications in the field of electrical technology, industrial automation, intelligent control and communication, and has important practical significance (X.N. Lu et al, 2012). There are many researches on phase measurement, which are many methods, such as zero checking method, FFT measurement phase method and zero-IF quadrature demodulation method. Zero checking method is simple, the calculation is small, and the measuring speed is fast. It is a simple and easy phase detection method. But its antiinterference ability is poor, especially for second harmonic, the results of phase detection by a lot of factors, resulting in phase detection accuracy is not high (Y.R. Liang, 2013). FFT phase measurement method is the digital phase measurement is often used means, but FFT method need other corrective measures were measured by means of, when the sampling frequency and FFT frequency interval non integer relationship, non-synchronous sampling of the phase measurement is not accurate and fast Fourier transform (FFT) exist obvious spectral leakage problems, the phase measurement accuracy decreases (X. Feng et al, 2008) (Z.H. Wang et al, 2007). Zero-IF quadrature demodulation method can not only deal with the low frequency signal, but also can realize the phase measurement of the high frequency signal through the down conversion technology. 
This method not only can measure the phase shift but also can measure the amplitude attenuation (X.F. $\mathrm{Hu}, 2006)$, selection of zero-IF quadrature demodulation method is used to measure the characteristics of medium voltage power line channel. Its schematic diagram is shown in Fig.1. The channel shape of heat accumulator is square and its cross section is shown in Fig.1.

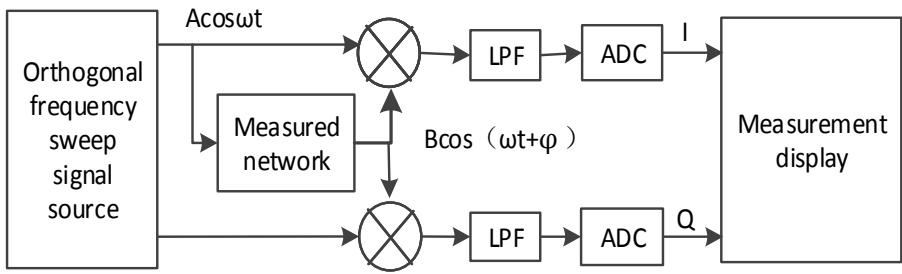

Fig.1 Zero-IF quadrature demodulation measurement principle diagram

Assuming $A \cos \omega t$ is the input signal for the test network, Asin $\omega t$ is the corresponding orthogonal signal. The amplitude attenuation and phase shift of the input signal after being measured, $B \cos (\omega t+\varphi)$ is the output.

Signal through the multiplier:

$A \cos \omega t \cdot B \cos (\omega t+\varphi)=\frac{A B}{2} \cos (2 \omega t+\varphi)+\frac{A B}{2} \cos \varphi$

$A \sin \omega t B \cos (\omega t+\varphi)=\frac{A B}{2} \sin (2 \omega t+\varphi)-\frac{A B}{2} \sin \varphi$

After low pass filtering, high frequency signals containing $2 \omega t$ are filtered out, only $\frac{A B}{2} \cos \varphi$ and $\frac{A B}{2} \sin \varphi$ associated with the offset phase $\varphi$ are left. This is not only the amplitude attenuation information but also the phase shift information. Through the A/D transform to obtain the corresponding $I$ and $Q$ values.

By using the trigonometric function to obtain amplitude attenuation of $\alpha$ :

$I^{2}+Q^{2}=\left(\frac{A B}{2} \cos \varphi\right)^{2}+\left(\frac{A B}{2} \sin \varphi\right)^{2}=\left(\frac{A B}{2}\right)^{2}$

The values of $I$ and $Q$ can be measured, the value of the amplitude $A$ of the input signal is known, according to the equation, we can get the amplitude $B$.

So the amplitude attenuation:

$\alpha=20 \lg \frac{B}{A}(d B)$

The equation for phase shift:
$\frac{Q}{I}=\tan \varphi \Rightarrow \varphi=\arctan \frac{Q}{I}$

The traditional zero-IF quadrature demodulation method is used to measure the local signal, its measurement is done in the same place. For medium voltage power line, transmission distance is relatively far. In actual measurement, it is unable to change the position and topology of the power line. The method described above is not feasible. As to solve the problem of long distance phase measurement, this paper presents a zero-IF quadrature demodulation method based on GPS. Its principle is shown in Fig.2.

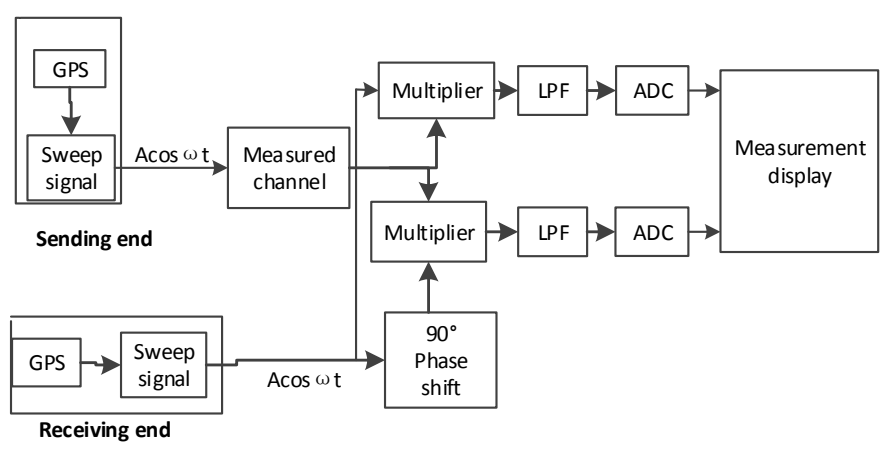

Fig.2 Quadrature demodulation phase measurement method based on GPS remote synchronization

The principle is as follows: Using two characteristics of the same signal source, placing on both ends of the tested network. When the measurement, through the GPS, controlling two signal source at the same time output the same frequency and phase of the signal. As long as the synchronization between the two signal source is high enough, it can be regarded as the same as the output of a single signal source. The principle of the second half is the same as the zero-IF quadrature demodulation method introduced above. To achieve high accuracy of synchronization is the key to the realization of the measurement method.

\section{CONCRETE REALIZATION PROCESS}

GPS 1PPS second pulse timing accuracy can reach dozens of nanoseconds, using GPS synchronization, two signal source in a 1PPS second pulse rising edge while the output, can meet the requirements of measurement precision. The specific implementation process is as follows:

A. After it starts, at the sending end, GPS module output 1PPS second pulse rising edge triggered the sending end CPU, CPU generated synchronization sequence and output, the synchronization sequence is modulated after sending, then the sending end turned receive mode. 
B. After the start, the receiving end has been in receive mode, when receives the synchronization sequence, receiving end CPU to check, if error check without subsequent operations, continue to in receive mode waiting to receive; if the verification is correct returns response sequence, and in a GPS 1PPS second pulse rising edge control sweep frequency signal generator output.

C. If after transmitting a synchronization sequence, the next 1PPS second pulse rising edge before the arrival, the sending end fails to receive or receives the wrong response sequence from the receiving end, then in the next 1PPS second pulse rising along the arrival time to send synchronization sequence; if the sending end correct indeed received the receiving end returns the response sequence, in the next 1PPS second pulse rising along the arrival time control sweep frequency signal generator generates a frequency sweep signal (sine or cosine signal).

D. After the receiving end sends response sequence, then it receives sequence synchronization again, it indicates that the last synchronization process failed, and needs to synchronize again, this time receiving end CPU retry response sequence, and wait for the next 1PPS second pulse rising edge to control of the sweep frequency signal generator output again.

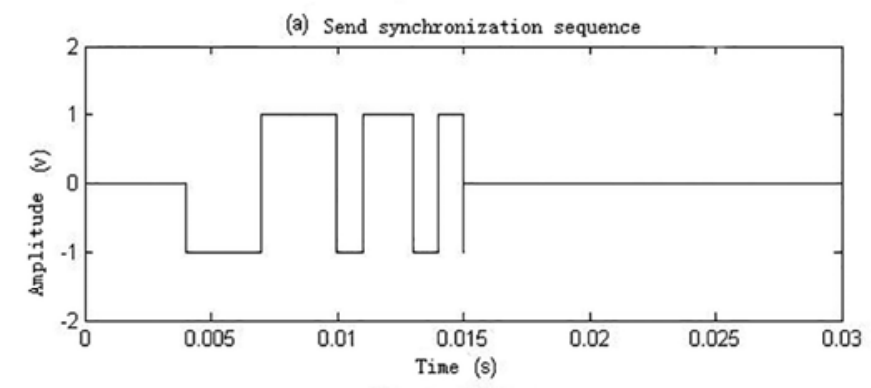

(c) Receive synchronization sequence

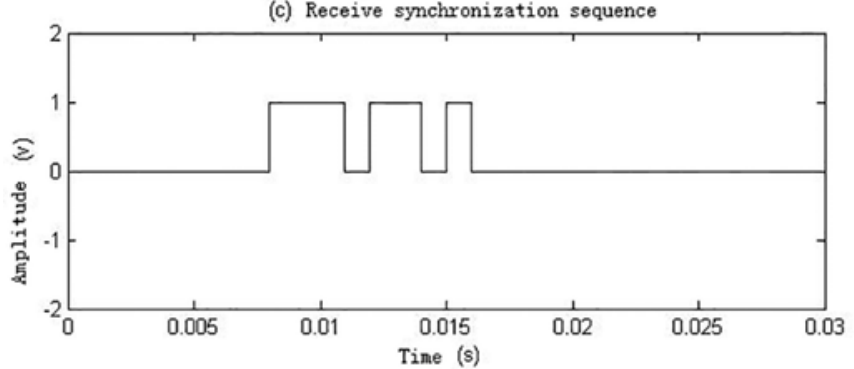

(e) Synchronous sequence checking

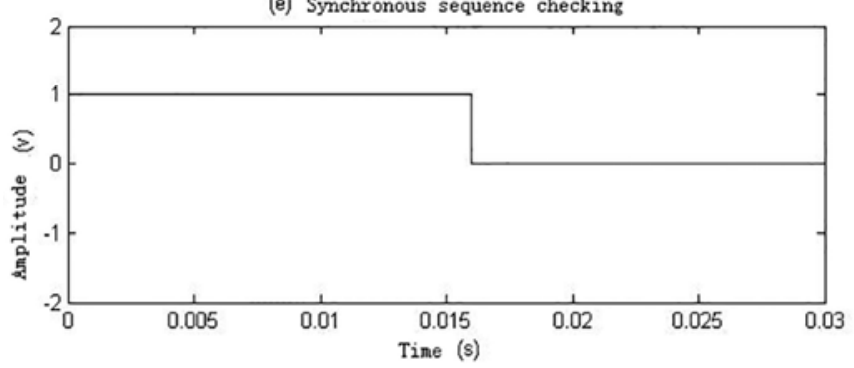

E.When all the synchronization process is correct, the receiving end receives the measured signal from the sending end. Then, using the quadrature demodulation phase measurement method to process the received measurement signal and the output signal from the receiving end sweep signal generator. Finally, calculating and displaying the measured results of phase shift and amplitude attenuation.

\section{SIMULATION RESULTS AND ANALYSIS}

In order to verify the correctness and feasibility of the method, the Simulink part of MATLAB is used to simulate and verify.

\subsection{Simulation of ideal channel synchronization and phase measurement.}

Simulation of synchronization and phase measurement is carried out on the condition of ideal channel. Using the rising edge of the pulse signal to simulate the GPS receiver output pulse rising edge of 1PPS second, after running the fourth clock cycles start synchronization, the synchronous sequence code is 00011101101, the answer sequence code is 0001101, Sweep signal for 2 seconds, the output frequency from $10 \mathrm{~Hz}$ to $400 \mathrm{kHz}$, amplitude is $1 \mathrm{~V}$. The simulation results are shown in Fig.3 and Fig.4.
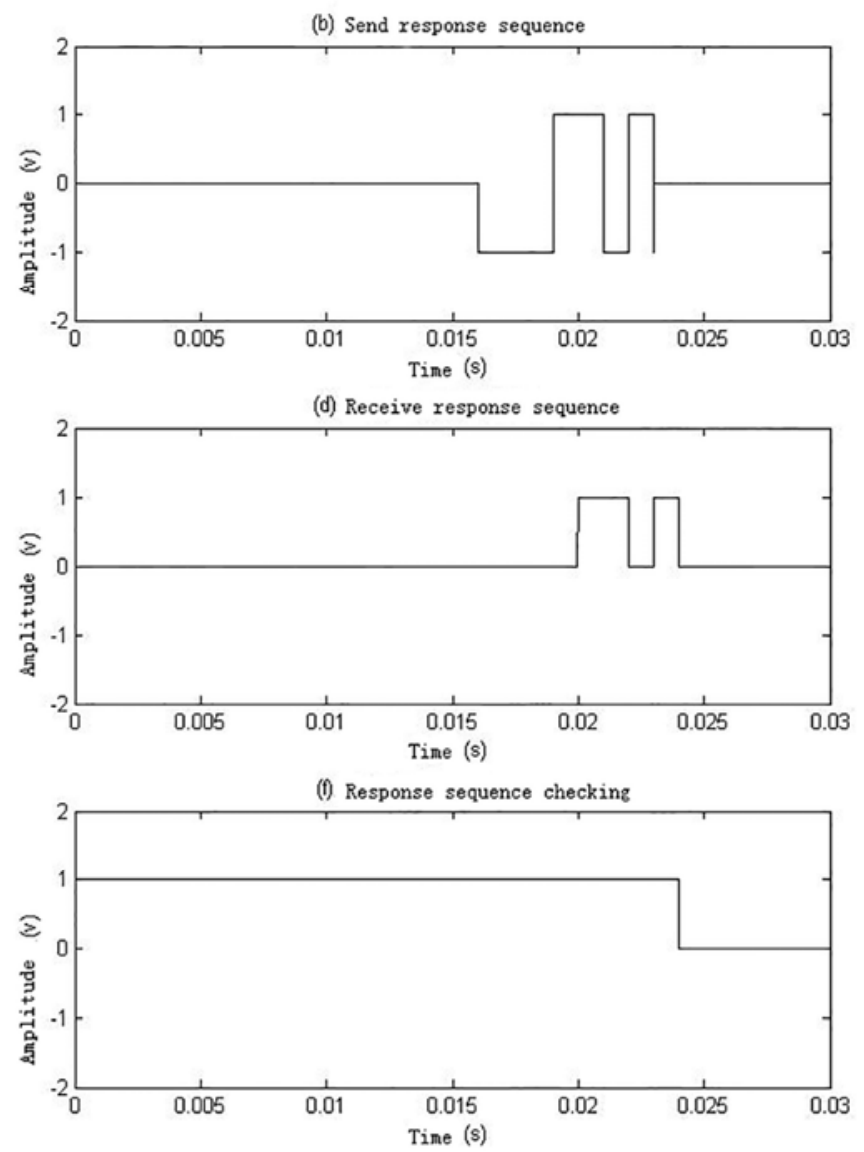

Fig.3 Simulation results of ideal channel synchronization 
(a) Phase measurement

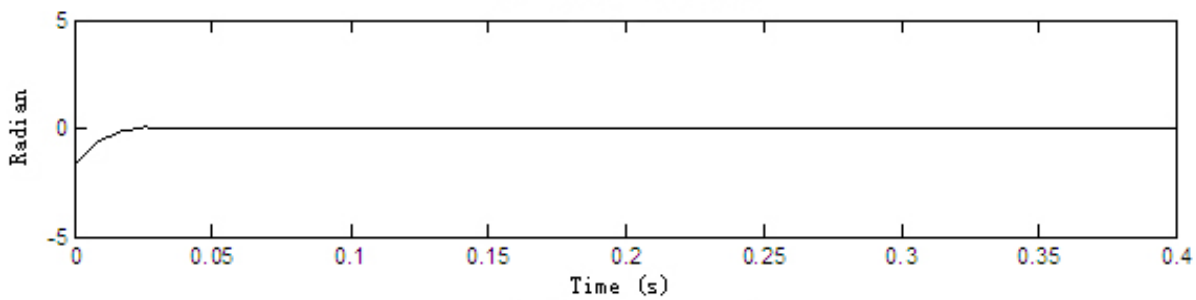

(b) Phase measurement

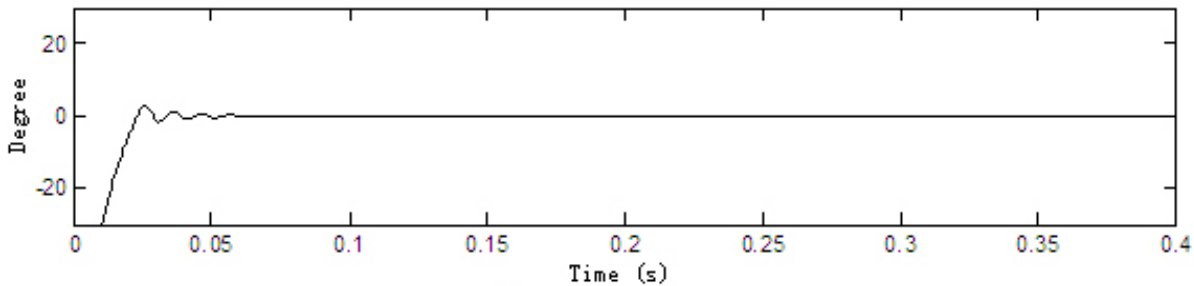

(c) Amplitude measurement

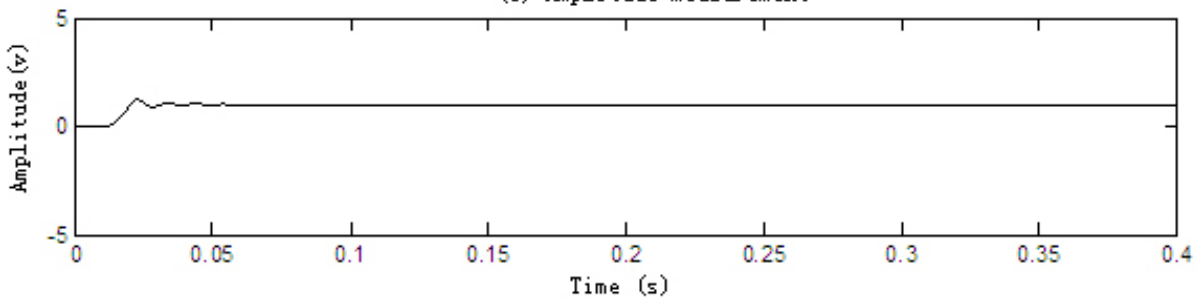

Fig.4 Simulation results of ideal channel phase measurement

Can be seen from Fig.3, synchronization sequence output at the start of the fourth clock cycle, after a clock cycle of the transmission delay, the synchronization sequence begins to receive. After the check is correct, return the response sequence, then receive reply sequence and verify correct.

In Fig.4, the results are stable after a short convergence process. The phase shift and amplitude attenuation of the simulation results are zero, which is consistent with the practical characteristics of the ideal channel.

(a) Send spuchronization sequence

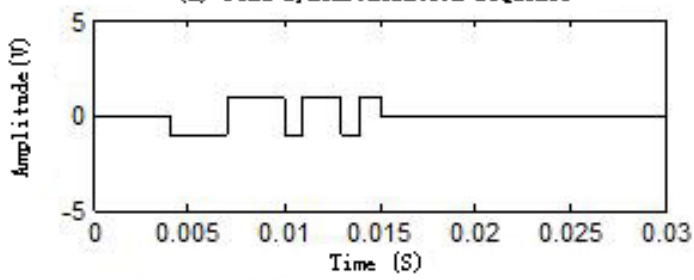

(c) Receive spuchronization sequence

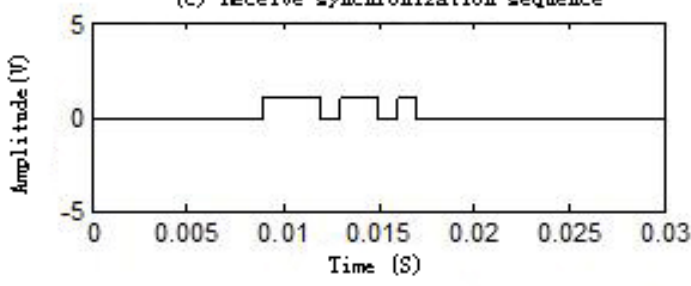

(e) Antolitude measurement

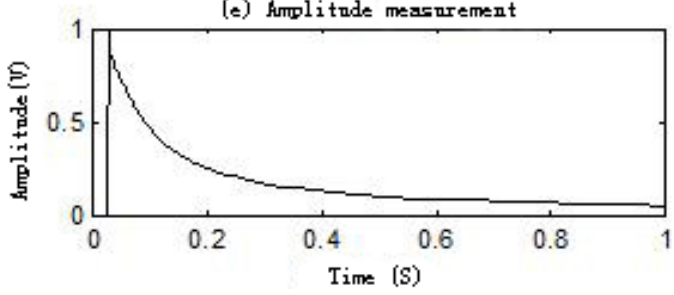

The simulation results show that the synchronization process and the phase measurement results are correct in the ideal channel condition. The method of zero-IF quadrature demodulation based on GPS remote synchronization is feasible.

\subsection{Simulation of channel synchronization and phase measurement in $R C$ circuit.}
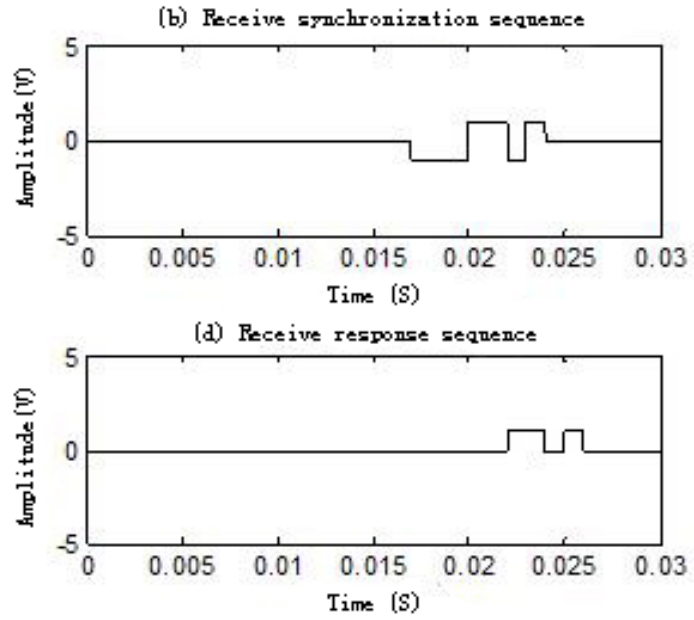

(f) Phase measurement

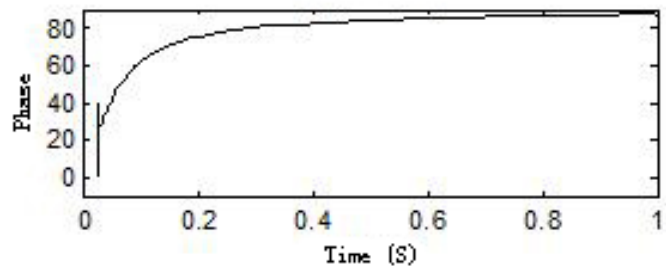

Fig.5 Simulation results of RC circuit channel system 
In order to further verify the zero-IF quadrature demodulation method based on GPS remote synchronization, the RC circuit is used as a channel, the amplitude attenuation and phase shift characteristics of the channel are known. Sweep signal for 2 seconds, the output frequency from $10 \mathrm{~Hz}$ to $400 \mathrm{kHz}$, amplitude is $1 \mathrm{~V}, \mathrm{RC}$ circuit adopts low pass mode, resonance frequency is $10.402 \mathrm{kHz}$, the other parameter settings are the same as that of the ideal channel simulation. The simulation results are shown in Fig.5.

As can be seen from Figure 6, the channel characteristics are measured at the beginning of the synchronization of the system, and the simulation results are consistent with the characteristics of the RC circuit. When the resonant frequency phase shift is 45 degrees, with the increase of frequency, the amplitude gradually tends to zero, the phase gradually tends to 90 degrees, but the amplitude attenuation curve does not show the process of changing from the convex function to the concave function, the transition point is the resonant frequency point. Analysis of the reasons is due to the resonant frequency settings over the entire frequency range is too small relative, makes the convex function component less than resonance frequency component unable to displayed. So we change the resonant frequency to $104.0228 \mathrm{kHz}$, sweep signal for 8 seconds, the output frequency from $10 \mathrm{~Hz}$ to $800 \mathrm{kHz}$. The simulation results are shown in Fig.6.

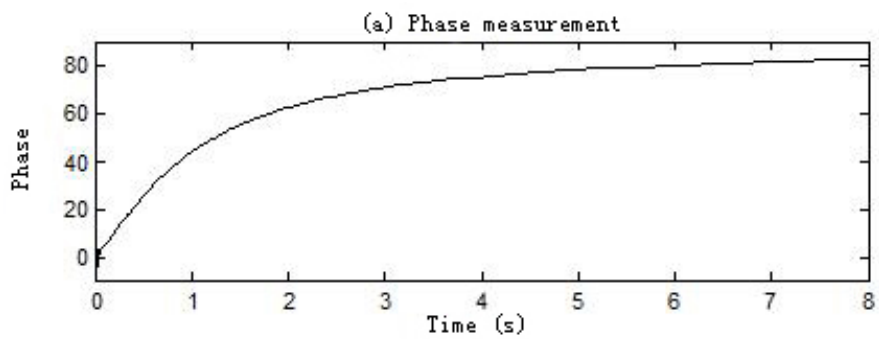

(b) Amplitude measurement

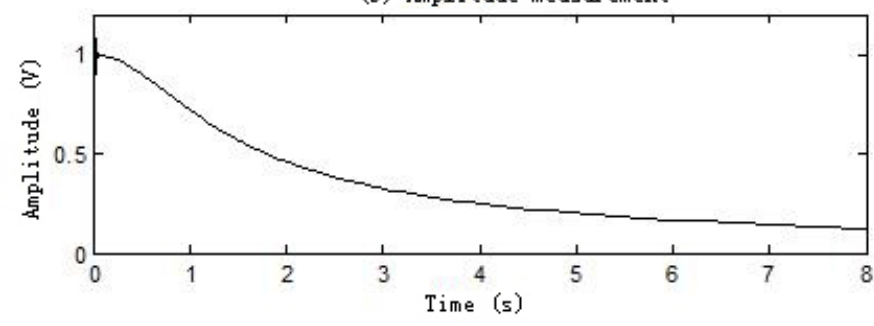

Fig. 6 The simulation results of the RC circuit after changing the resonant frequency

As can be seen from Fig.7, the measurement results of the phase shift and amplitude attenuation characteristics of the RC circuit are consistent with the actual RC circuit characteristics. The correctness and feasibility of the zero-IF quadrature demodulation method based on GPS remote synchronization is proved.

\section{CONCLUSION}

In order to solve the phase measurement of the long distance channel of the medium voltage power line, this paper proposes a zero-IF quadrature demodulation phase measurement method based on GPS remote synchronization. In this paper, the principle and implementation process are introduced in detail, and the simulation results are consistent with the actual situation of the ideal channel and RC channel. The correctness and feasibility of this method is verified by the simulation results.

\section{ACKNOWLEDGEMENTS}

This work was supported by "National Science Foundation of China” (No.61172075).

\section{REFERENCES}

S. Xu, H.Y. Yi and X.Y. Wang: Power System Communication, Vol.31 (2010) No. 207, p. 52.

X. Feng, G. Zhao: National Conference of electrical testing technology (Hangzhou, China, October, 2008).

X.F. Hu: Study on beam position and phase measurement of $A D S$ proton linear accelerator, (Ph.D., Yanshan University, China 2006).

X.N. Lu, Y.H. Zhang: Engineering and Technology Institute of the United Kingdom (Stevenage, England, 2012).

Y.R. Liang: High precision phase measurement in heterodyne laser interferometer (Ph.D., Huazhong University of Science and Technology, China 2013).

Z.H. Wang, X.D. Huang and W. Yang: World Science and Technology Research and Development, Vol.29 (2007) No. 4, p. 28. 